\title{
Case Report of Neonatal Proteus mirabilis Meningitis and Brain Abscess with Negative Initial Image Finding: Consideration of Serial Imaging Studies
}

Mi Hae Chung, Geonju Kim, Aluem Han, and Juyoung Lee

Department of Pediatrics, College of Medicine, The Catholic University of Korea, Seoul, Korea

\section{ABSTRACT}

Proteus mirabilis (P. mirabilis) meningitis in a neonate is rare, but its recognition is important because the disease progresses rapidly and has poor prognosis. A 4-dayold premature female infant born at 36 weeks and 5 days of gestation presented with symptoms of fever and icteric skin. Initial cerebrospinal fluid findings suggested bacterial meningitis, and treatment with antibiotics was started. On the third day, $P$. mirabilis growth was found in both blood and cerebrospinal fluid cultures and brain computed tomography revealed normal findings. The patient showed improved clinical symptoms, but brain magnetic resonance imaging on hospital day 18 revealed a brain abscess measuring $4.5 \times 3.1 \times 3.1 \mathrm{~cm}$ in the right frontal lobe. Cyst drainage was performed immediately and a catheter was inserted. Follow-up computed tomography revealed a tiny abscess remaining in the right frontal lobe, and follow-up magnetic resonance imaging later demonstrated marked interval regression in the size of the abscess. The patient was discharged on day 57 of hospitalization in good condition. Serial brain imaging should be considered in neonatal cases of $P$. mirabilis meningitis.

Key Words: Proteus mirabilis, Meningitis, Brain abscess, Newborn

\section{INTRODUCTION}

Few cases of neonatal Proteus mirabilis (P. mirabilis) meningitis have been reported in the literature. Most patients exhibited poor feeding, lethargy, and fever within several days after birth as the initial symptoms, and rapid progression resulting in severe neurological sequelae or death. In addition to initial studies to identify neonatal sepsis or meningitis, brain imaging studies are important because progression of this disease can occur even after normalization of cerebrospinal fluid (CSF) findings through the use of antibiotics ${ }^{1,2}$. Here, we report a neonatal case of $P$. mirabilis meningitis in which the initial brain imaging findings were normal, but a brain abscess was found in a later follow-up study.
Received: 8 September 2017

Revised: 24 October 2017

Accepted: 25 October 2017

Correspondence to: Juyoung Lee Department of Pediatrics, Bucheon St. Mary's Hospital, College of Medicine, The Catholic University of Korea, 327 Sosa-ro, Bucheon 14647, Korea

Tel: +82-32-340-7022

Fax: +82-32-340-2314

E-mail: frocner@catholic.ac.kr

Copyright(c)

By Korean Society of Neonatology.

All right reserved.

This is an Open-Access article distributed under the terms of the Creative Commons Attribution Non-Commercial License (http://creativecommons.org/licenses/ by-nc/4.0), which permits unrestricted non-commercial use, distribution, and reproduction in any medium, provided the original work is properly cited. 


\section{CASE REPORT}

A 4-day-old premature female infant born at 36 weeks and 5 days of gestation by spontaneous delivery with a birth weight of $3.44 \mathrm{~kg}$ was referred to our hospital showing symptoms of fever and icteric skin. Fever started on the day of admission. The mother had history of recurrent cystitis, but the urine analysis during pregnancy was normal and she did not show symptoms relevant to cystitis throughout her pregnancy. The antenatal, intrapartum, and immediate postnatal periods were uneventful, and there was no reported premature rupture of the membrane or symptoms suggestive of infection such as fever.

The infant's peripheral white blood cell (WBC) count was 19,300 cells $/ \mu \mathrm{L}$ with a differential count of $74.4 \%$ neutrophils, $10.1 \%$ lymphocytes, $15.4 \%$ monocytes, and $0.1 \%$ basophils. The hemoglobin was $15.0 \mathrm{~g} / \mathrm{dL}$, and the platelet count was 236,000 cells $/ \mu \mathrm{L}$. Urine analysis findings were negative, and sediment examination revealed 1-4 WBCs/high-power field (HPF) and 1-4 red blood cells/HPF. The initial CSF analysis revealed leukocytosis (corrected WBC count $1,589 / \mathrm{mm}^{3}$ ) with a predominance of neutrophils (82\%) and increased protein level (146 mg/dL), which suggested bacterial meningitis. An initial course of antibiotics comprising cefotaxime and vancomycin was started at doses targeted for meningitis.

On hospital day 2, the growth of gram-negative bacilli in blood culture was found, and the antibiotics were changed to cefotaxime and amikacin. On hospital day 3 , growth of $P$. mirabilis, which is susceptible to cefotaxime, amikacin, and meropenem, was found in both blood and CSF cultures. The urine culture results were negative. The patient showed slight tonic movements suggestive of seizure activity and phenobarbital loading was started. A second CSF analysis showed a markedly decreased

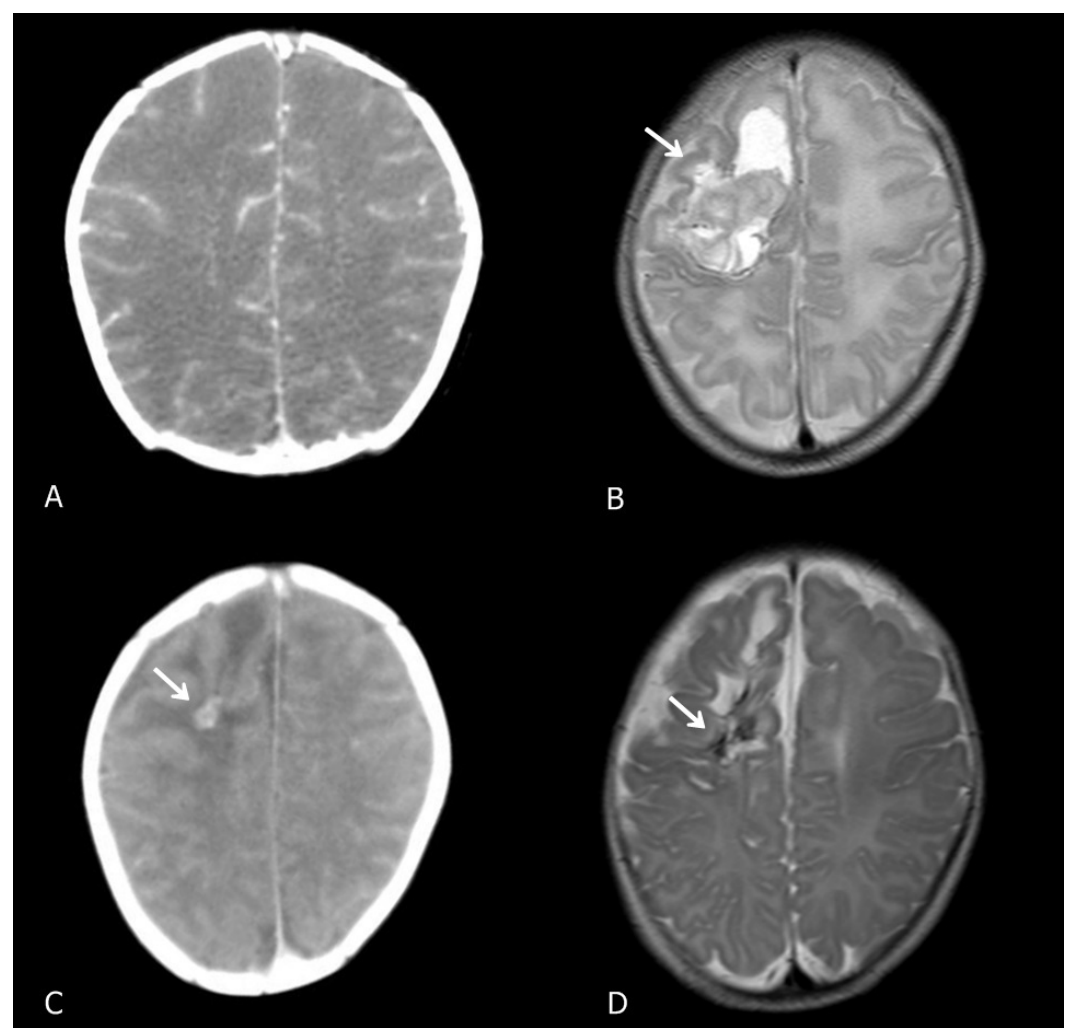

Figure 1. Brain imaging findings in chronological order: (A) initial brain CT shows negative findings on HD 3; (B) follow-up brain MRI (T2WI, enhanced) reveals brain abscess in right frontal lobe (arrow) on HD 18; (C) follow-up brain CT shows a tiny abscess remaining in the right frontal lobe (arrow) on HD 42; and (D) second follow-up brain MRI (T2WI, enhanced) shows marked interval regression in the size of the abscess (arrow) on HD 55. Abbreviations: CT, computed tomography; HD, hospital day; MRI, magnetic resonance imaging; T2WI, T2-weighted image. 
WBC count (corrected count $52 / \mathrm{mm}^{3}$ ), and brain computed tomography (CT) showed normal findings (Figure 1A). The patient's fever subsided on hospital day 4 and no seizure-like activity was observed afterward. On hospital day 13, electroencephalography (EEG) showed sharp waves occurring in the right frontocentral and occipital areas, which indicated focal seizures. Because no clinical symptoms were observed, the phenobarbital was stopped. However, the patient showed tonic-clonic movements the next day, and phenobarbital treatment was resumed. Brain magnetic resonance imaging (MRI) was performed on hospital day 18 and showed a mass measuring $4.5 \times 3.1 \times 3$.1 $\mathrm{cm}$ in the right frontal lobe with lobulated rim enhancement and internal septation (Figure 1B). A catheter was inserted for immediate cyst drainage. The drained fluid was yellowish-green in color and exhibited a high WBC count although no bacteria were cultured. Cefotaxime and amikacin were continued for 3 more weeks; the total antibiotic treatment lasted 6 weeks. No seizure activity was observed after drainage.

A follow-up brain CT exam was performed on hospital day 42 and showed a tiny abscess remaining in the right frontal lobe and progression of leukomalacic changes in both frontal lobes and in the genu of the corpus callosum (Figure 1C). Because of the remaining abscess, the antibiotics were changed to meropenem. A follow-up MRI on hospital day 55 showed a remnant encephalomalacic lesion containing a small rim-enhancing nodular lesion in the right frontal lobe, which indicated marked interval regression of the abscess (Figure 1D). The patient was discharged on day 57 of hospitalization in good condition.

\section{DISCUSSION}

This appears to be the first neonatal case of $P$. mirabilis menin- gitis reported in Korea. Case reports of $P$. mirabilis meningitis in neonates have mainly reported poor outcomes, including death or neurological impairment. Imaging studies are essential for detecting and monitoring $P$. mirabilis meningitis because this disease can lead to purulent meningitis and ventriculitis ${ }^{1,3)}$, brain abscess $^{2,4)}$, hydrocephalus ${ }^{2)}$, extensive pneumocephalus ${ }^{5)}$, global anoxic injury $^{6)}$, or hemorrhagic meningoencephalitis ${ }^{7}$. In our patient, brain CT was performed on day 3 of admission, which was 2 days after symptom onset, and showed normal findings. The patient's clinical symptoms also improved; that is, her fever subsided and her seizures were controlled with phenobarbital. Thus, it was difficult to predict that a brain MRI on day 18 of admission would reveal a large brain abscess. Our experience suggests that, in cases of $P$. mirabilis, delayed progression of brain lesions may develop even without apparent clinical symptoms. A case reported by Smith and Mellor ${ }^{2)}$ in 1980 showed a similar clinical course in a term newborn treated for $P$. mirabilis meningitis. Brain imaging was not performed, and the infant was discharged at age 27 days with improved clinical symptoms. However, the infant was later readmitted at age 36 days because of irritability and was found to have bilateral frontal abscess formation ${ }^{2}$.

Imaging findings of six neonatal cases of $P$. mirabilis meningitis are listed in Table $1^{2-7)}$. In the cases reported by Kassim et al. ${ }^{5)}$ and Juyal et al. ${ }^{3)}$, CT could not be performed on day 1 of admission because of the patients' unstable condition. Such circumstances are expected because $P$. mirabilis meningitis often progress rapidly and is accompanied by severe symptoms such as apnea, desaturation, hypotonia, and convulsion. In cases in which imaging studies were performed immediately on the day of admission, abnormalities such as global anoxic damage and hemorrhagic meningoencephalitis were noted. Brain abscesses were found when the imaging was performed at least a week after disease onset. The finding of a brain abscess on brain MRI imaging on

Table 1. Reported Neonatal Cases of Proteus mirabilis Meningitis

\begin{tabular}{lcccc}
\hline Case author, year & Symptom onset age & $\begin{array}{c}\text { Age at hospital } \\
\text { admission }\end{array}$ & $\begin{array}{c}\text { Initial imaging } \\
\text { (age/ HD) }\end{array}$ & Imaging finding \\
\hline Smith et al., 1980) & 24 hours & 2 days & CT (37 days/HD 36) & Bilateral frontal abscess \\
Kassim et al., 2003 & 2 days & 5 days & CT (10 days/HD 5) & Extensive pneumocephalus \\
Phan and Lehman, 2012 & 10 days & 11 days & CT (17 days/HD 7) & Left frontal abscess \\
Juyal et al., 2013 & 2 days & 5 days & CT (10 days/HD 12) & Meningoventriculitis \\
Omoruyi and Evangelista, 2014 & 4 days & 6 days & CT (6 days/HD 1) & Global anoxic injury \\
Coskun et al., 2017 & 4 days & 6 days & MRI (6 days/HD 1) & Hemorrhagic meningoencephalitis \\
\hline
\end{tabular}

Abbreviations: CT, computed tomography; HD, hospital day; MRI, magnetic resonance imaging. 
hospital day 18 in our patient is consistent with the results of these earlier studies.

Two cases involved serial imaging ${ }^{4,7)}$, but this was not possible in other cases because the patients had died. In the case reported by Phan and Lehman, the initial brain CT performed 7 days after the onset of symptoms revealed a left frontal cavitary lesion. Follow-up MRI performed 8 and 19 days after symptom onset showed a more clearly defined brain abscess, and the final MRI at 40 days after symptom onset showed near complete resolution of the abscess ${ }^{4}$. In the case reported by Coskun et al., hemorrhagic meningoencephalitis was seen on the initial brain MRI performed 2 days after symptom onset, and follow-up MRI studies at 1.5 and 4 months of age showed intermediate and late phase changes of hemorrhagic meningoencephalitis, which led to severe cerebral atrophy and encephalomalacia ${ }^{7}$. Our case is unique in showing a negative initial image finding on CT (performed 2 days after symptom onset) but later appearance of a brain abscess on follow-up MRI 17 days after symptom onset.

According to Parmar and Ibrahim, brain abscess formation caused by cerebritis occurs in four different stages: early cerebritis, late cerebritis, early capsule formation, and late capsule formation $^{8)}$. Infection is not localized during the initial stage of early cerebritis (3-5 days), and focal necrotic areas are formed and encapsulation is started during the late cerebritis stage (514 days). A well-defined capsule is found in the early and late capsule formation stages (weeks to months) ( $^{8}$.

A normal CT scan is the most common image finding in children with acute bacterial meningitis ${ }^{8)}$. Contrast-enhanced T1weighted MRI is the standard method of imaging for intracranial meningitis, and contrast-enhanced fluid attenuation inversion recovery imaging increases the specificity for detection of subtle leptomeningeal enhancement ${ }^{8)}$. Thus, brain MRI is recommended for initial imaging in cases involving bacterial meningitis. However, in our case, this was not possible because of the patient's unstable condition, and thus, we chose to perform a CT scan, which takes less time. The CT scan was negative when performed 2 days after symptom onset, which means that the brain abscess may not have been detected because it was in the early cerebritis stage. Inclusion of an initial MRI study may have shown findings related to acute bacterial meningitis.

The initial choices of antibiotics are mainly ampicillin or penicillin with a combination of aminoglycosides ${ }^{2-7)}$. Antibiotics can be changed to chloramphenicol, cefotaxime, and meropenem if the patient's condition deteriorates ${ }^{5}$, fever persists despite initial antibiotic use ${ }^{4)}$, seizure occurs ${ }^{7}$, or when the antibiotics do not match the culture sensitivities ${ }^{2}$. For our patient, we chose cefotaxime and vancomycin as initial antibiotic empirical treatment for bacterial meningitis. When the blood culture showed growth of gram-negative bacilli, we changed the antibiotics to cefotaxime and amikacin, which were continued for 6 weeks. The antibiotic treatment was later changed to meropenem because of concern about the remaining brain abscess observed on the follow-up CT.

Gram-negative bacillary meningitis is associated with a higher risk of mortality and morbidity ${ }^{9)}$ and with an increased incidence of brain abscess formation when caused by species such as Citrobacter diversus ${ }^{10)}$ and Enterobacter sakazakii ${ }^{11)}$. Clinicians treating patients with $P$. mirabilis meningitis should be aware of this high risk. We have examined the progression of neonatal cases of $P$. mirabilis meningitis reported in the literature to try to identify the optimal time for brain imaging. However, the number of cases is too small and the course of the disease too variable to run an analysis. Future studies of $P$. mirabilis meningitis are needed to understand better the clinical progression of the disease. Our case indicates that serial imaging studies should be considered in neonatal cases of $P$. mirabilis meningitis, even in patients with improved clinical symptoms.

\section{REFERENCES}

1) Levy HL, Ingall D. Meningitis in neonates due to Proteus mirabilis. Am J Dis Child 1967;114:320-4.

2) Smith ML, Mellor D. Proteus mirabilis meningitis and cerebral abscess in the newborn period. Arch Dis Child 1980;55:308-10.

3) Juyal D, Rathaur VK, Sharma N. Neonatal meningoventriculitis due to proteus mirabilis - a case report. J Clin Diagn Res 2013;7: 369-70.

4) Phan H, Lehman D. Cerebral abscess complicating Proteus mirabilis meningitis in a newborn infant. J Child Neurol 2012; 27:405-7.

5) Kassim Z, Aziz AA, Haque QM, Cheung HA. Isolation of Proteus mirabilis from severe neonatal sepsis and central nervous system infection with extensive pneumocephalus. Eur J Pediatr 2003;162:644-5.

6) Omoruyi EA, Evangelista M. Proteus mirabilis septicemia and meningitis in a neonate. J Med Cases 2014;5:245-7.

7) Coskun Y, Akman I, Yildirim C, Demir MK. A newborn with hemorrhagic meningoencephalitis due to Proteus mirabilis. Drug Discov Ther 2017;10:334-7. 
8) Parmar H, Ibrahim M. Pediatric intracranial infections. Neuroimaging Clin N Am 2012;22:707-25.

9) Kim KS. Acute bacterial meningitis in infants and children. Lancet Infect Dis 2010;10:32-42.
10) Graham DR, Band JD. Citrobacter diversus brain abscess and meningitis in neonates. JAMA 1981;245:1923-5.

11) Willis J, Robinson JE. Enterobacter sakazakii meningitis in neonates. Pediatr Infect Dis J 1988;7:196-9. 